\title{
The Impact of Amplitude-Integrated Electroencephalography on NICU Practice
}

\author{
Juan Pablo Appendino, Patrick J. McNamara, Matthew Keyzers, Derek Stephens, \\ Cecil D. Hahn
}

\begin{abstract}
Objective: To examine how the introduction of amplitude-integrated electroencephalography (aEEG) to our neonatal intensive care unit (NICU) influenced clinical practice. Methods: This was a retrospective study examining clinical practice three years before and three years after the introduction of aEEG monitors to our NICU. A time series analysis was performed to explore whether aEEG introduction was associated with changes in the rates of conventional EEGs performed, neurology consultations and neonates diagnosed with seizures. Results: Following aEEG introduction, the total number of conventional EEGs performed remained constant; however, there was significant shift in conventional EEG utilization towards neonates receiving fewer multiple EEGs and more single EEGs. There was no change in the rate of neurology consultations or the number of neonates diagnosed with seizures. Conclusions: Introduction of aEEG monitors to our NICU has led to less reliance on conventional EEG as a tool for the serial evaluation of brain function. Since the number of neonates diagnosed with seizures did not increase, aEEG monitoring did not appear to uncover a significant subgroup of patients with subclinical seizures that would previously have gone undetected. Conventional EEG and aEEG are complementary tools for the assessment of newborn cerebral function.
\end{abstract}

RÉSUMÉ: Impact de l'électroencéphalographie à amplitude intégrée sur la pratique à l'unité néonatale de soins intensifs. Objectif : Le but de l'étude était d'examiner comment l'introduction de l'électroencéphalographie à amplitude intégrée (EEGa) dans notre unité néonatale de soins intensifs (UNSI) a influencé la pratique clinique. Méthode : Nous avons procédé à une étude rétrospective de la pratique clinique au cours des trois années qui ont précédé l'introduction de moniteurs EEGa dans notre UNSI et des trois années qui ont suivi son implantation. Nous avons effectué une analyse de séries chronologiques pour évaluer si l'introduction de l'EEGa était associée à un changement dans les taux d'EEG conventionnels effectués, de consultations en neurologie et de diagnostic de crises convulsives chez les nouveaux-nés. Résultats : Suite à l'implantation de l'EEGa, le nombre total d'EEG conventionnels effectués est demeuré constant. Cependant, nous avons observé un changement important dans l'utilisation de l'EEG conventionnel chez les nouveaux-nés qui ont subi moins d'EEG multiples et plus d'EEG uniques. Il n'y a eu aucun changement dans le taux de consultations en neurologie ou dans le nombre de nouveaux-nés chez qui on a diagnostiqué des crises convulsives. Conclusions : Suite à l'installation de moniteurs EEGa dans notre UNSI, nous avons noté que l'EEG conventionnel était moins utilisé comme outil pour l'évaluation en série de la fonction cérébrale. Comme le nombre de nouveaux-nés chez qui un diagnostic de crises convulsives n'a pas augmenté, la surveillance EEGa ne semble pas avoir détecté un sous-groupe important de patients qui présentent des crises subcliniques qui n'auraient pas été détectées antérieurement. L'EEG conventionnel et l'EEGa sont des outils complémentaires dans l'évaluation de la fonction cérébrale chez le nouveau-né.

Can J Neurol Sci. 2012; 39: 355-360

Amplitude-integrated electroencephalography (aEEG), also termed cerebral function monitoring (CFM) is a bedside brain monitoring tool that has gained widespread acceptance in neonatal intensive care units (NICUs) around the world. aEEG technology is based on a 1- or 2-channel EEG signal transformed into a time-compressed depiction of the range of EEG amplitudes, providing an overview of trends in cerebral background activity and the occurrence of seizures. Applications of aEEG have included selecting candidates for therapeutic hypothermia ${ }^{1}$, evaluating severity of injury and cerebral recovery after a hypoxic-ischemic insult ${ }^{2}$, predicting neurodevelopmental outcome $\mathrm{e}^{3-6}$, and diagnosing clinical and subclinical seizures ${ }^{7-8}$.

Despite the widespread adoption of aEEG in many NICUs, the diagnostic utility of aEEG and its role in relationship to conventional EEG recordings remains a matter of continued debate ${ }^{7-10}$. Several studies have demonstrated good agreement between aEEG and conventional EEG in evaluating background activity and detecting electrographic seizures, with the exception of focal, low amplitude or brief ( $<30$ second) seizures, which are often missed on aEEG. ${ }^{7,11,12}$ Some experts have recommended using aEEG as a monitoring device and performing intermittent

From the Division of Neurology (JPA, CDH), Division of Neonatology (PJM, MK), Clinical Research Support Unit (DS), The Hospital for Sick Children Research Institute and University of Toronto, Toronto, Ontario; Division of Neurology (JPA), Children's Hospital of Winnipeg and the University of Manitoba, Winnipeg, Manitoba, Canada.

Received August 29, 2011. Final Revisions Submitted November 3, 2011.

Correspondence to: Cecil D. Hahn, Division of Neurology, The Hospital for Sick

Children, 555 University Avenue, Toronto, Ontario, M5G 1X8, Canada.

Email: cecil.hahn@sickkids.ca 
conventional EEG whenever there is any doubt about the classification of the $\mathrm{aEEG}^{11}$.

Despite the extensive literature comparing the specificity and sensitivity of aEEG versus conventional EEG for background classification and seizure detection, little is known about the impact of aEEG on conventional EEG utilization and NICU practice. The objective of this study was to investigate how the introduction of aEEG has affected NICU clinical practice at our institution. Specifically, we examined whether the introduction of aEEG was associated with changes in the frequency and timing of conventional EEG recordings, the frequency of neurology consultations, and the rates of diagnosis of neonatal seizures.

\section{METHODS}

The goal of this population-based NICU study was to evaluate changes in overall clinical practice before and after the aEEG introduction to our NICU. This was a retrospective study of all neonates admitted to the NICU at our institution during a six year period, divided into two epochs: the three years prior to the introduction of aEEG (Epoch 1: July 2001-June 2004) and the three years after introduction of aEEG (Epoch 2: July 2004June 2007). An analysis of how conventional EEG and aEEG influenced the care of individual patients was beyond the scope of this study, since the retrospective data set limited our ability to ascertain the rationale for individual clinical decisions.

The study was approved by the institutional Research Ethics Board with a waiver of informed consent. The following data were abstracted from the electronic patient chart, health records database, and clinical databases of all conventional EEG and
aEEG recordings: the frequency and time of initiation of both conventional EEG and aEEG recordings in the NICU, the number of EEG recordings performed on each patient, the number of neurology consultations performed in the NICU, and the number of neonates diagnosed with seizures. Neonates diagnosed with seizures were identified based on the following ICD-10 discharge diagnoses (or their ICD-9 equivalents): G40 (epilepsy), G41 (status epilepticus), P90 (convulsions of newborn) and R56 (convulsions, not elsewhere classified). We also documented the following covariables: the monthly rate of NICU admissions, and the monthly average Score for Neonatal Acute Physiology - Perinatal Extension II (SNAPPE-II) ${ }^{13}$ for all NICU admissions, as a measure of overall illness severity.

\section{aEEG monitoring}

The first aEEG machine was introduced to our NICU in July 2004, and in the intervening period (June 2005 and a third in November 2005) two additional devices have been added. aEEG recordings were performed by trained NICU respiratory therapists using either a 1-channel (Olympic CFM 6000, Natus Medical Inc., San Carlos, CA) or 2-channel system (Brainz BRM 2, Natus Medical Inc., San Carlos, CA). Recording electrodes for the 1-channel system were applied at C3 and C4, whereas for the 2-channel system they were placed $1 \mathrm{~cm}$ anterior and posterior to $\mathrm{C} 3$ and C4. Amplitude-integrated electroencephalography recordings were routinely available $24 / 7$, with electrode application performed by in-house respiratory therapists. The aEEG monitoring was requested by the NICU medical team according to clinical practice guidelines. The clinical indications for aEEG recordings were hypoxic-

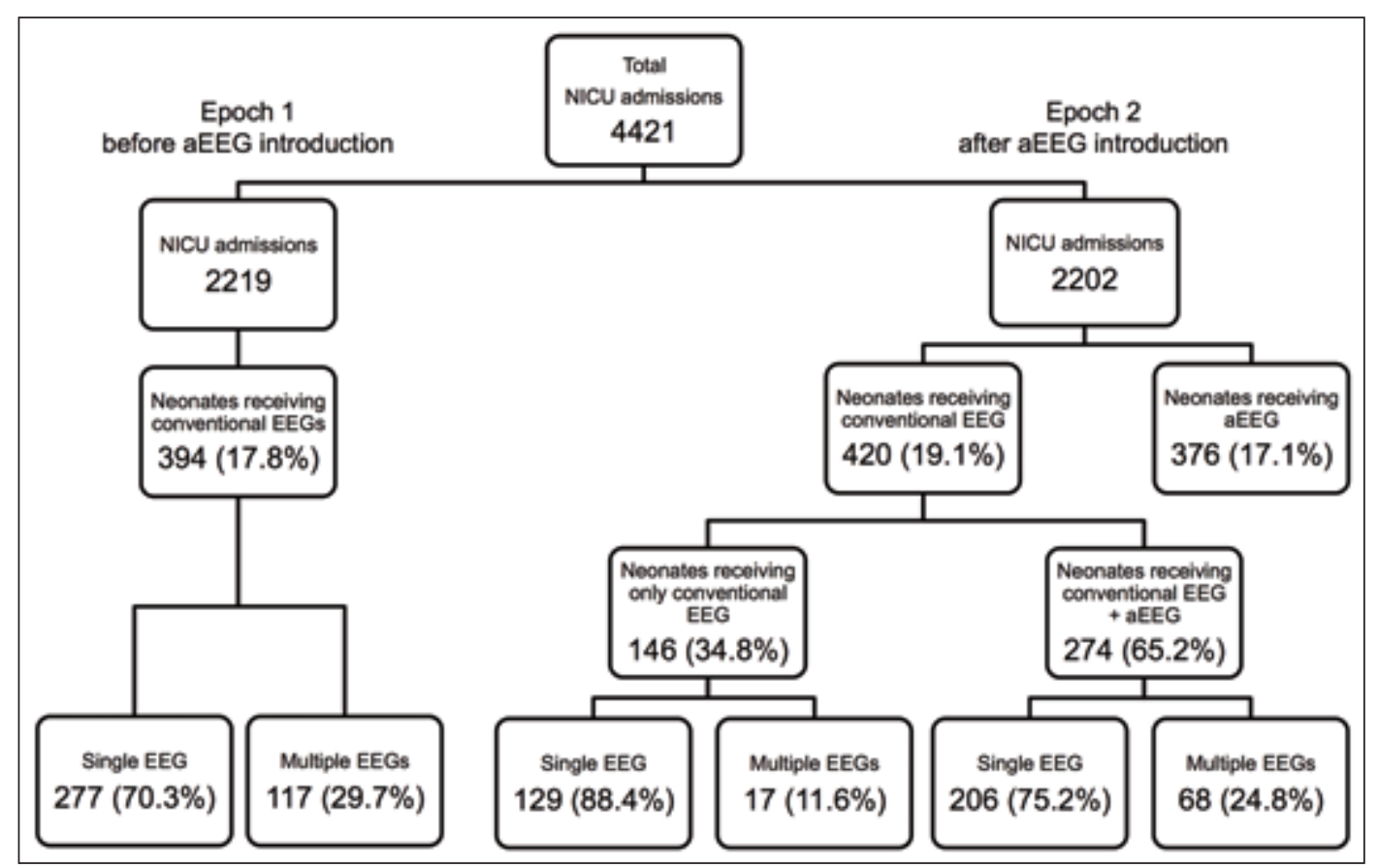

Figure 1: Frequency of NICU admissions, and neonates receiving conventional EEG and aEEG recordings before (Epoch 1) and after (Epoch 2) the introduction of aEEG. 


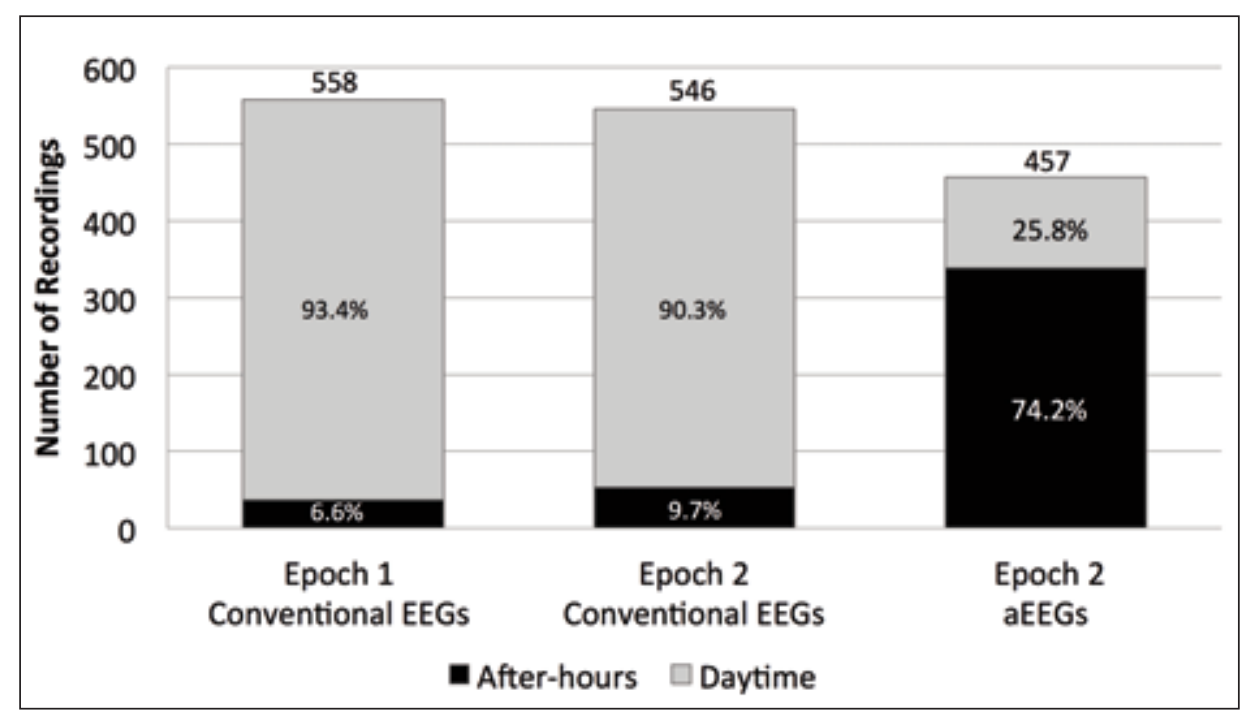

Figure 2: Frequency of conventional EEG and aEEG recordings performed during daytime and afterhours, before (Epoch 1) and after (Epoch 2) introduction of aEEG.

ischemic encephalopathy, seizures or suspected seizures (e.g., apnea, episodic hypertension or tachycardia), a significant neurologic disorder (e.g., congenital brain malformations, stroke), cardiac arrest, inborn errors of metabolism (e.g., urea cycle disorders, hypoglycaemia, hypocalcemia) and neonatal abstinence syndrome (e.g., alcohol/opiate withdrawal). aEEG recordings are interpreted by in-house NICU physicians and nursing staff at the bedside, who have received three hours of introductory didactic and hands-on teaching, followed by regular learning activities as part of routine clinical care and continuing medical education.

\section{Conventional EEG}

In contrast, conventional EEG recordings were performed by board-certified EEG technologists and interpreted by a team of neurologists and neurophysiologists. Conventional EEG recordings were requested at the discretion of the NICU medical team or the Neurology consult team, the indications being seizures or suspected seizures and the assessment of neonatal encephalopathy. Conventional EEG recordings were performed by registered EEG technologists using a neonatal variant of the international 10-20 system for durations between 30 and 60 minutes ${ }^{14}$. Conventional EEG studies were routinely available during regular daytime working hours (Monday to Friday from 08:00 to 16:30 hours), and EEG technologists could be called in after-hours for urgent requests (Monday to Fridays 16:30 to 08:00 hours, plus weekends and holidays). The availability of conventional EEG recordings remained constant before and after the introduction of aEEG in our NICU. Conventional EEG recordings were interpreted on the day of recording by boardcertified neurophysiologists.

\section{Statistical Analysis}

Univariate analyses were performed using paired t-tests to test for significant differences in the average monthly rates of each of the above variables and covariables before and after the introduction of aEEG. Time series analysis, using the above variables summarized by calendar month of admission, was then used to investigate whether the introduction of aEEG was associated with a change in the frequency and timing of conventional EEG recordings, a change in the number of neurology consultations, or a change in the number of neonates diagnosed with seizures. An indicator term was used to model the three year epochs before and after introduction of aEEG. Time series analyses were adjusted for the monthly rate of NICU admissions and the average monthly SNAPPE-II scores. Since all series investigated were found to be stationary with nonsignificant autocorrelation at all lags (Ljung-Box test), a generalized linear model using a Poisson distribution was additionally explored on all series. Statistical significance was set at $\mathrm{p}<0.05$. All analyses were performed using SAS v9.1.2 (SAS Institute, Inc., Cary, NC).

\section{RESULTS}

The study population comprised 4421 neonates, equally divided between Epoch 1 (before aEEG introduction) and Epoch 2 (after aEEG introduction). The number of neonates receiving conventional EEG, aEEG or both tests is depicted in Figure 1.

Although there was some month-to-month variability, the rates of NICU admissions and conventional EEGs performed remained relatively stable over the six year study period. Following the introduction of the first aEEG machine in July 2004, the monthly rate of aEEG recordings increased rapidly to approximate the rate of monthly conventional EEGs performed. Introduction of a second and third aEEG machines was not associated with a change in the monthly rate of aEEGs performed; however, there was a significant increase in the duration of aEEG recordings (mean of 23.0 hours during the first six months of epoch 2 versus 33.4 hours during the last six months of epoch 2, $\mathrm{p}=0.02$ ) Over $90 \%$ of conventional EEG 


\begin{tabular}{|c|c|c|c|}
\hline \multirow[b]{2}{*}{ Variable } & \multicolumn{2}{|c|}{ Mean Monthly Rates (SD) } & \multirow[b]{2}{*}{$\mathrm{p}$-value } \\
\hline & Before aEEG & After aEEG & \\
\hline Number of Admissions to NICU & $61.6(6.4)$ & $61.2(7.7)$ & 0.78 \\
\hline Mean SNAPPE scores & $11.1(2.9)$ & $9.3(2.3)$ & $<0.01$ * \\
\hline Conventional EEGs performed in NICU & $15.5(4.7)$ & $15.2(6.0)$ & 0.79 \\
\hline Daytime & $14.5(4.4)$ & $13.7(5.4)$ & 0.51 \\
\hline After-hours & $1.0(1.1)$ & $1.5(1.7)$ & 0.20 \\
\hline Neonates receiving conventional EEGs & $11.1(3.7)$ & $11.6(4.9)$ & 0.61 \\
\hline Single EEG & $7.8(3.2)$ & $9.3(4.4)$ & 0.11 \\
\hline Multiple EEGs & $3.3(1.7)$ & $2.3(1.7)$ & 0.02 * \\
\hline Neurology Consultations & $6.2(2.5)$ & $6.1(3.5)$ & 0.91 \\
\hline Neonates Diagnosed with Seizures & $6.3(2.2)$ & $6.6(2.8)$ & 0.58 \\
\hline
\end{tabular}

SD: standard deviation; $* \mathrm{p}<0.05$ on Student's t-test

recordings were initiated on weekdays during daytime working hours (Figure 2). Following the introduction of aEEG there was a non-significant increase in the number of after-hours conventional EEG recordings (from $6.6 \%$ to $9.7 \%, \mathrm{p}=.08$ ). In contrast, $74 \%$ of aEEG recordings were initiated after-hours or on weekends.

Univariate analyses (Table 1) demonstrated that although the monthly rate of neonates receiving conventional EEG did not change following aEEG introduction, the rate of neonates receiving multiple EEGs declined significantly (3.3 vs. 2.3 per month, $\mathrm{p}=0.02$ ). The average rates of neurology consultations performed in the NICU, and neonates diagnosed with seizures did not change following aEEG introduction. There was no significant difference in the number of NICU admissions between the two epochs, but mean SNAPPE-II scores were significantly lower during Epoch 2 . Time series analysis (Table 2), adjusted for monthly admission rates and SNAPPE-II scores, revealed that while the overall rate of conventional EEG recordings performed in the NICU did not change following the introduction of aEEG, there was a significant increase in the rate of neonates receiving single EEGs (by 2.18 neonates/month; $\mathrm{p}<0.01$ ), and a concomitant decrease in the rate of neonates receiving multiple EEGs (by 0.78 neonates/month; $\mathrm{p}=0.02$ ). Again, there was no significant change in the rates of neurology consultations or neonates diagnosed with seizures.

\section{DiscuSSION}

This study demonstrates that the introduction of aEEG to the NICU at our institution was not associated with a significant change in the overall frequency of conventional recordings, however, there was a significant change in the pattern of conventional EEG usage: fewer neonates received multiple EEGs, and more neonates received single EEGs. We speculate that this shift in conventional EEG utilization reflects the combination of two shifts in NICU practice in response to aEEG availability. First, the availability of aEEG as a continuous monitoring tool may have reduced the need for serial conventional EEGs to document the temporal evolution of EEG background activity required for accurate prognostication. Second, availability of aEEG may have led to an increased use of conventional EEGs as a confirmatory diagnostic test, to evaluate suspected abnormalities detected during aEEG monitoring. Interestingly, the rate of neurology consultations to the NICU remained stable at an average of six consults per month, suggesting that the availability of aEEG did not alter neurology consultation practices in our NICU.

The introduction of aEEG to our NICU did not result in an increase in the diagnosis of neonatal seizures. Given that the majority of neonatal seizures detected by conventional EEG are known to be subclinical ${ }^{15,16}$ and given that $15 \%$ of neonates experiencing seizures may have purely subclinical seizures ${ }^{17}$, one might have expected aEEG to uncover a subgroup of neonates with subclinical seizures that would have previously gone undetected. The fact that rates of neonatal seizure diagnosis remained stable in our NICU may reflect the lower sensitivity of aEEG for seizure detection compared with conventional $\mathrm{EEG},{ }^{7,18}$ or may simply reflect a low rate of neonates with purely subclinical seizures among population. A previous study has demonstrated poor sensitivity of aEEG for detection of brief $(<$ 90 seconds) or focal seizures ${ }^{7}$.

The difference in timing of conventional EEG vs aEEG initiation was striking. Seventy-four percent of aEEG recordings were initiated after-hours compared to $10 \%$ of conventional EEGs. In fact, the proportion of aEEG recordings initiated afterhours corresponds to the proportion of evening and weekend hours $(75 \%)$ that fall outside of a typical 40-hour workweek, reflecting the 24/7 in-house availability of respiratory therapists and medical staff responsible for aEEG initiation and trace 


\begin{tabular}{|c|c|c|}
\hline Variable & $\begin{array}{l}\text { Change in monthly rate following } \\
\text { aEEG introduction }(95 \% \mathrm{Cl})\end{array}$ & $\mathrm{p}$-value \\
\hline Conventional EEGs performed in NICU & $0.74(-1.03,2.50)$ & 0.42 \\
\hline Daytime & $0.29(-0.83,1.41)$ & 0.61 \\
\hline After-hours & $0.44(-0.73,1.62)$ & 0.46 \\
\hline \multicolumn{3}{|l|}{ Neonates receiving conventional EEGs } \\
\hline Single EEG & $2.18(1.38,2.99)$ & $<0.01$ * \\
\hline Multiple EEGs & $-0.78(-1.39,-0.17)$ & $0.02 *$ \\
\hline Neurology Consultations & $-0.11(-0.58,0.35)$ & 0.64 \\
\hline Neonates Diagnosed with Seizures & $0.72(-0.14,1.58)$ & 0.11 \\
\hline
\end{tabular}

SD: standard deviation; $* \mathrm{p}<0.05$ on Student's t-test

interpretation. Although conventional EEG recordings were also available 24/7, after-hours requests required a neurology consultation and calling in the EEG technologist from home. Interestingly, once aEEG became available in our NICU, afterhours requests for conventional EEG did not decline. Rather, there was a non-significant trend towards more after-hours conventional EEG use (6.6\% of conventional EEGs before vs. $9.7 \%$ after aEEG introduction), possibly reflecting an increased interest in brain monitoring, or the desire to confirm aEEG findings on an urgent basis.

Our findings support the notion that conventional and amplitude-integrated EEG are complementary tools for the assessment of newborn cerebral function. Contrary to the expectation of some neurologists, the introduction of aEEG to our NICU has not supplanted conventional EEG: $72.8 \%$ of newborns monitored with aEEG also underwent conventional EEG recording. Rather, the two techniques appeared to play different roles. Given that aEEG was available 24/7 in-house, neonatologists often appeared to rely on aEEG for after-hours assessments, and conventional EEG during daytime hours, perhaps as a confirmatory tool. This appears to be a reasonable strategy in the absence of $24 / 7$ availability of full-montage continuous video-EEG monitoring. Interestingly however, following the introduction to our NICU in 2008 of continuous full-montage video-EEG monitoring with real-time remote review, the majority of continuous brain monitoring in our NICU is still conducted using 1- or 2-channel aEEG machines (data not shown). This suggests that our neonatologists have a continued preference for aEEG over continuous video-EEG recordings, possibly because of greater familiarity with the bedside aEEG display, or greater ease of access to aEEG since this service is provided by in-house NICU personnel.

This study has limitations. Our observations on the impact of aEEG introduction may not be representative of other similar tertiary care institutions, due to institutional differences in the working relationship between neonatologists and neurologists, the availability of conventional EEG recordings or other factors.
Although our analyses controlled for illness severity, there may have been other secular trends in neonatal practice that are unaccounted for, and may have biased our results. Because our data on the diagnosis of neonatal seizures were based on ICD-10 coding, we were unable to examine the proportion of clinical versus subclinical seizures. Diagnostic criteria for neonatal seizures were not standardized in our NICU. Thus, there may have been differences in diagnostic approach between attending physicians; however, the attending physicians working in our NICU remained largely the same over the course of the study, limiting the potential for bias. Finally, because this study employed a population-level analysis, we could not examine the impact of aEEG on the management of individual patients. A prospective study would be required to measure the impact of aEEG on clinical decision-making at an individual patient level and neonatal outcomes, because the rationale for clinical decision making is difficult to ascertain from a retrospective review of patient charts.

We conclude that aEEG introduction in our NICU led to less reliance on conventional EEG as a tool for serial evaluation of brain function; but this is offset by an increase in requests for a single conventional EEG, which we hypothesize is to confirm abnormalities detected on aEEG. Since the number of neonates diagnosed with seizures did not increase, aEEG monitoring did not appear to uncover a significant subgroup of patients with subclinical seizures that would previously have gone undetected. The effect of aEEG on individual clinical decision-making such as seizure management requires further prospective study.

\section{AKNOWLEDGEMENTS}

This work was supported a research grant from The Hospital for Sick Children Foundation. The authors thank Loreto Lecce, NICU Information Systems Manager, and Debi Senger, Donnette Newman, Majid Kiakojouri, and Veronica Harris from the Department of Health Records for the invaluable assistance. 


\section{Disclosures Or CONFlict Of INTERest}

Matthew Keyzers reports that he has acted as an educational consultant for Natus Medical, Inc.

\section{REFERENCES}

1. Gluckman PD, Wyatt JS, Azzopardi D, et al. Selective head cooling with mild systemic hypothermia after neonatal encephalopathy: multicentre randomised trial. Lancet. 2005; 365: 663-70.

2. al Naqeeb N, Edwards AD, Cowan FM, Azzopardi D. Assessment of neonatal encephalopathy by amplitude-integrated electroencephalography. Pediatrics. 1999; 103: 1263-71.

3. Hellstrom-Westas L, Rosen I, Svenningsen NW. Predictive value of early continuous amplitude integrated EEG recordings on outcome after severe birth asphyxia in full term infants. Arch Dis Child Fetal Neonatal Ed. 1995; 72: F34-8.

4. ter Horst HJ, Sommer C, Bergman KA, Fock JM, van Weerden TW, Bos AF. Prognostic significance of amplitude-integrated EEG during the first 72 hours after birth in severely asphyxiated neonates. Pediatr Res. 2004; 55: 1026-33.

5. Shany E, Goldstein E, Khvatskin S, et al. Predictive value of amplitude-integrated electroencephalography pattern and voltage in asphyxiated term infants. Pediatr Neurol. 2006; 35 : 335-42.

6. Toet MC, Hellstrom-Westas L, Groenendaal F, Eken P, de Vries LS. Amplitude integrated EEG 3 and 6 hours after birth in full term neonates with hypoxic-ischaemic encephalopathy. Arch Dis Child Fetal Neonatal Ed. 1999; 81: F19-23.

7. Shellhaas RA, Soaita AI, Clancy RR. Sensitivity of amplitudeintegrated electroencephalography for neonatal seizure detection. Pediatrics. 2007; 120: 770-7.

8. Mathur AM, Morris LD, Teteh F, Inder TE, Zempel J. Utility of prolonged bedside amplitude-integrated encephalogram in encephalopathic infants. Am J Perinatol. 2008; 25: 611-15.
9. Freeman JM. The use of amplitude-integrated electroencephalography: beware of its unintended consequences. Pediatrics. 2007; 119: 615-17.

10. Shah DK, de Vries LS, Hellstrom-Westas L, Toet MC, Inder TE. Amplitude-integrated electroencephalography in the newborn: a valuable tool. Pediatrics. 2008; 122: 863-5.

11. Hellström-Westas L. Comparison between tape-recorded and amplitude-integrated EEG monitoring in sick newborn infants. Acta Paediatr. 1992; 81: 812-19.

12. Toet MC, van der Meij W, de Vries LS, Uiterwaal CS, van Huffelen KC. Comparison between simultaneously recorded amplitude integrated electroencephalogram (cerebral function monitor) and standard electroencephalogram in neonates. Pediatrics. 2002; 109: 772-9.

13. Richardson DK, Corcoran JD, Escobar GJ, Lee SK. SNAP-II and SNAPPE-II: simplified newborn illness severity and mortality risk scores. J Pediatr. 2001; 138: 92-100.

14. Guideline 2: Minimum technical standards for pediatric electoencephalography. J Clin Neurophysiol. 2006; 23: 92-6.

15. Clancy RR, Legido A, Lewis D. Occult neonatal seizures. Epilepsia. 1988; 29: 256-61.

16. Murray DM, Boylan GB, Ali I, Ryan CA, Murphy BP, Connolly S. Defining the gap between electrographic seizure burden, clinical expression and staff recognition of neonatal seizures. Arch Dis Child Fetal Neonatal Ed. 2008; 93: F187-91.

17. Scher MS, Alvin J, Gaus L, Minnigh B, Painter MJ. Uncoupling of EEG-clinical neonatal seizures after antiepileptic drug use. Pediatr Neurol. 2003; 28: 277-80.

18. Bourez-Swart MD, van Rooij L, Rizzo C, et al. Detection of subclinical electroencephalographic seizure patterns with multichannel amplitude-integrated EEG in full-term neonates. Clin Neurophysiol. 2009; 120: 1916-22. 